Die Zukunft der Nachhaltigkeitsberichterstattung II/IV

\section{Weg von der Erbsenzählerei, hin zu den wesentlichen Fragen}

\author{
In Teil II der Serie hinterfragt Heike Leitschuh den Sinn \\ standardisierter Vergleichbarkeit von Nachhaltigkeitsberichten. \\ Die Philosophie und Haltung des Unternehmens müsse in \\ ihnen erkennbar sein. Der ideale Nachhaltigkeitsbericht ist für \\ sie kurz, aber gehaltvoll. Von Heike Leitschuh
}

W e könnten, wie sollten Nachhaltigkeitsberichte aussehen, damit sie für die Leser(innen) wirklich nützlich sind? Und vor allem auch für das Unternehmen. Denn, wie wir wissen, ist die Erstellung eines solchen Berichts auch ein intensiver Prozess der Diskussion und Verständigung über die aktuelle Situation, den künftigen Weg und die Ziele des Unternehmens. Nicht selten werden bei diesem Prozess erst viele Schwachstellen offenkundig, aber auch unerkannte Potenziale entdeckt. Allein das hartnäckige Beharren, dem sich die verschiedenen Unternehmensbereiche ausgesetzt sehen, Daten und Fakten beizutragen, führt oft dazu, dass dort ein zunehmendes Verständnis für die Belange der Nachhaltigkeit entsteht.

Aber sagen wir besser: Das ist nur dann so, wenn der Prozess nicht schematisch aufgesetzt ist und wenn nicht schon zu viel Routine im Spiel ist. Die Richtlinien der Global Reporting Initiative sind sicher hilfreich, doch besteht die Gefahr, dass Berichterstattung zur seelenlosen Managementfleißaufgabe verkommt: Vor lauter Zahlen und Fakten kann man nicht (mehr) erkennen, was der Sinn, die Philosophie, die Haltung der Unternehmung und vor allem der sie prägenden Menschen sind.

\section{Auf künftige Heraus- forderungen einstellen}

Standardisierte Vergleichbarkeit ist gut und richtig, aber worauf kommt es bei der Berichterstattung wirklich an? Wollen wirklich alle im Detail wissen, wie sich der Wasserverbrauch eines Unternehmens an den verschiedenen Standorten entwickelt hat, auch wenn Wasser nicht das zentrale Nachhaltigkeitsthema dieser Firma ist? Oder geht es nicht vielmehr darum zu erfahren, ob und wie sie sich auf die aktuellen und künftigen Herausforderungen einstellt, die am Standort, national wie international, absehbar sind? Ich bevorzuge Letzteres.

Ein idealer Nachhaltigkeitsbericht umfasst daher für mich nicht viel mehr als 20 Seiten - diese aber gehaltvoll; alle detaillierten Daten und Fakten (vor allem für den Kapitalmarkt und für die Steuerungsfähigkeit des Unternehmens) gehören ins Internet und zwar verbindlich für alle Unternehmen. In dem Bericht sollte das Unternehmen vor allem zu drei Themenbereichen Stellung nehmen:

- Wie sieht unser Geschäftsmodell aus? Wie bewerten wir es vor dem Hintergrund der gesellschaftlichen Herausforderungen und absehbaren Veränderungen? Sind unsere Produkte und Dienstleistungen die richtigen auf dem Weg in eine nachhaltige Gesellschaft? Sind sie auch in fünf oder zehn Jahren noch die richtigen? An welchen Stellen sind wir uns sicher, dass wir das Richtige tun, an welchen Stellen haben wir Zweifel? Was tun wir, um mehr Klarheit zu bekommen?

- Stand der Zielerreichung: Was hatten wir uns vorgenommen? Was haben wir seit dem letzten Bericht erreicht?
Was nicht? Warum hat nicht alles geklappt? Mit welchen Dilemmata haben wir es zu tun? Welche Zielkonflikte konnten wir nicht auflösen? Warum nicht? Was haben wir daraus gelernt?

- Künftige Ziele: Wo wollen wir hin? Was sind unsere kurz-, mittel- und langfristigen Ziele? Welche Maßnahmen scheinen geeignet? Wo sehen wir in Zukunft Probleme bei der Umsetzung? Welche Mittel erscheinen uns sinnvoll, um Zielkonflikte zu bearbeiten?

\section{Unternehmen trauen sich zu wenig}

Das sind aus meiner Sicht die wesentlichen Fragen an einen guten Nachhaltigkeitsbericht. Leider aber scheinen sich nur wenige Unternehmen zu trauen, über dieses „Eingemachte“ zu schreiben. Vielleicht gibt es dafür auch einen nachvollziehbaren Grund: Nicht nur die Konkurrenz, auch die zivilgesellschaftlichen Akteure und vor allem Medien werfen sich ja gerne genüsslich auf die Schwachstellen von Unternehmen. Dies tun sie jedoch vor allem dann, wenn das Unternehmen versucht, die wunden Punkte zu verbergen oder schönzureden.

Wirkliche Transparenz aber nimmt destruktiven Kritiker(inne)n den Wind aus den Segeln. Schließlich sind viele unternehmerische Zielkonflikte durchaus gesellschaftlich bedingt: Alle wollen superschnell, supersicher und superkomfortabel mit dem Zug reisen, aber gleichzeitig soll der Energieverbrauch und der Lärm sinken und die Zugtrassen sollen bitteschön nicht die Landschaft verschandeln.

Ich freue mich auf die Berichte, die so etwas zum Thema machen. Das wäre spannend.

I AUTORIN + KONTAKT

Heike Leitschuh ist Buchautorin, Moderatorin und Beraterin und arbeitet seit über 20 Jahren zu den Themen der Nachhaltigkeit. Sie ist Mitherausgeberin des „Jahrbuch Ökologie“.

Heike Leitschuh, Münchener Str. 23, 60329 Frankfurt, Tel.: +496970791284 E-Mail: heike-leitschuh@t-online.de Internet: www.fairwirtschaften.de 
Copyright (C) 2012, IÖW und oekom Verlag. Die Nutzung des Artikels ist Abonnenten von Ökologisches Wirtschaften vorbehalten. Nachdruck und Vervielfältigung des Artikels einschließlich Speicherung und Nutzung auf optischen und elektronischen Datenträgern nur mit Zustimmung der Redaktion von Ökologisches Wirtschaften (http://www.oekologischeswirtschaften.de). 